\title{
Preface
}

\section{Michael Hobday}

How best to understand, analyse and interpret the firm? This is not a trivial question. It goes to the heart of understanding the new (and old) economy. However, until now we have not yet had an approach which deals with the firm as part of the environment in which it is born and grows and competes. Neoclassical economics treats the firm as an entity devoid of history and context, operating only to maximize profit in its competitive setting. The Penrosian resource-based view is a far superior way of looking at the business firm, showing how it evolves through the development of capabilities and the allocation of resources by management to profitable new activities. However, a weakness of the Penrosian perspective is that it focuses on the business firm and cannot explain its relationship with, and embeddedness within, the environment in which it operates and evolves. By contrast, the evolutionary perspective examines how the environment selects among firms, by rewarding some and punishing others. Yet, somehow, the evolutionary perspective does not really capture the central role of business firms in actually shaping the environment.

By bringing together elements of a radical new approach to the firm, based on the biological metaphor of the ecosystem, this book goes beyond the limits of existing theories and tries to show precisely how the modern business firm competes and cooperates within its market and technological environment - equally, it touches on how firms actually shape the environment through their deliberate actions and investments. This new approach, called digital business ecosystems (DBEs) also allows us to understand the way new digital technologies feed into the environment and shape the ways in which firms and groups of firms compete and cooperate.

This book presents a fascinating collection of papers from different perspectives all addressing the DBE. It is both highly original and groundbreaking in its achievements. From a theory point of view, the study argues that the concept of region and history fails to account for the fact that the firm is not merely a member of a single industry but a part of a business ecosystem which cuts across a variety of industries. The ecosystem is supported by enabling technologies and, more specifically, digital software which changes and shapes not only the internal structure of firms but the 
ways in which firms work together. While the new theory is closely linked and sympathetic to the evolutionary theory of the firm pioneered by Nelson and Winter, DBE is less concerned with firm selection and more interested in path dependency of organizational forms and the emergence of 'organizational genotypes'.

The work is also rich in empirical evidence. The chapter on regional development in Europe is particularly interesting as it attempts to conceptualize and explain the challenges posed by the huge numbers of small and medium-sized enterprises within the European DBE. Another chapter deals with how DBEs relate to other historical organizational forms, pointing to the fundamental tasks of all business organizations, namely coordination and cooperation. Within the international context the research shows that, in fact, very few major companies have developed DBE structures effective enough to harness the potential of 'global knowledge diversity', implying that a change in business leader mindset is needed before the benefits of DBEs are to be fully realized.

Evidence to interpret and examine the impact and implications of DBEs is provided from a wide range of developed and developing countries, including the European Union, China, India, Singapore and the USA. With the emergence of China and India as important global players it is fascinating to see, for example, how Indian software service organizations created a particular form of DBE with a network of external linkages which both 'scaled up' and sustained the growth of this industry. A chapter on China shows that this rapidly growing economy will soon force a fundamental strategic rethink on the way transnational firms choose to globalize through DBEs. A second chapter on China deals with precisely how the new information and communications technologies which underpin DBEs will affect China's entire development trajectory and the very nature of globalization as it is currently understood.

The book provides frameworks and methods for understanding DBEs, covering issues of intellectual property, costs and benefit assessment techniques and a series of other useful tools. Perhaps one limitation of the book is that it does not go very far in showing how leading firms actually construct the DBE within which they compete. The concepts of 'selforganization' and 'co-evolution' do not seem to capture this vital role of leading firms. However, there are some fascinating glimpses into this issue (for example the chapters on Cisco, IBM, Microsoft and Netscape). What these chapters suggest is needed is more in-depth research and conceptualization on how and why particular firms introduce the new technologies, build the infrastructures and stimulate the markets within which they then compete - and how these processes underpin the self-organization of the DBE. In fact, the strict biological notion of self-organization may be 
challenged by these new conceptualizations of DBEs in the book, which suggest the importance of leadership, decision making and 'conscious' human strategy and action on the evolution of the DBE. No doubt these issues will be taken up further by the authors in this exciting new research agenda.

In summary, this is a unique text rich with theory, novelty and significant new arguments and empirical data. It extends our understanding of the firm in many positive new ways and is a very welcome and important contribution to the field of innovation studies. 